\title{
Comparison of the acute effects on gas exchange of nasal ventilation and doxapram in exacerbations of chronic obstructive pulmonary disease
}

\author{
R M Angus, A A Ahmed, L J Fenwick, A J Peacock
}

\begin{abstract}
Background - Nasal intermittent positive pressure ventilation (NIPPV) is useful in exacerbations of chronic obstructive pulmonary disease (COPD) complicated by ventilatory failure. The effects of NIPPV were compared with those of the respiratory stimulant doxapram on gas exchange in patients with COPD and acute ventilatory failure.

Methods - Patients admitted with acute exacerbations of COPD and type 2 respiratory failure $\left(\mathrm{PaO}_{2}<8 \mathrm{kPa}\right.$ and $\mathrm{PaCO}_{2}$ $>6.7 \mathrm{kPa}$ ) who did not improve with conventional treatment were randomised to receive either NIPPV or intravenous doxapram. Blood gas tensions were monitored for four hours.

Results - In nine patients who received NIPPV the arterial $\mathrm{PaO}_{2}$ improved from a mean (SE) of $5.9(0.4) \mathrm{kPa}$ to a maximum of $8.1(0.6) \mathrm{kPa}$ which was maintained at four hours. Eight patients who received doxapram had a similar baseline $\mathrm{PaO}_{2}$ of $5.6(0.4) \mathrm{kPa}$ which rose to a maximum of $7.3(0.5) \mathrm{kPa}$ but this was not maintained at four hours. The improvement in $\mathbf{P a O}_{2}$ in patients on NIPPV was accompanied by a fall in $\mathrm{PaCO}_{2}$ but, in contrast, in those who received doxapram there was no improvement in $\mathrm{PaCO}_{2}$.

Conclusions - NIPPV may be more effective than doxapram in the management of acute ventilatory failure complicating COPD.

(Thorax 1996;51:1048-1050)
\end{abstract}

Keywords: respiratory failure, NIPPV, doxapram.

Department of Physiotherapy

L J Fenwick

Western Infirmary and Gartnavel General Hospital,

Glasgow G11 6NT, UK

Correspondence to: Dr A J Peacock.

Received 26 June 1995 Returned to authors 23 October 1995 Revised version received 26 March 1996 Accepted for publication 29 April 1996 anical ventilation via an endotracheal tube. Doxapram drives respiration, probably by stimulating peripheral chemoreceptors. ${ }^{23}$ This is helpful if there is suppression of central drive but, in the context of an acute exacerbation of chronic lung disease, the respiratory muscles may already be functioning at near maximal capacity. Intubation and full scale intermittent positive pressure ventilation is invasive, expensive, and it may be difficult to wean the patients off the ventilator. An effective alternative would be desirable.

Nasal intermittent positive pressure ventilation (NIPPV) has also been shown to improve arterial blood gas tensions in patients with COPD, ${ }^{45}$ but no comparison has been published of the effects of NIPPV and doxapram in the emergency treatment of ventilatory failure. We have therefore compared NIPPV and doxapram in a group of patients presenting to a respiratory ward with type 2 respiratory failure.

\section{Methods}

PATIENTS

Patients were included if they had an acute exacerbation of COPD complicated by type 2 respiratory failure $\left(\mathrm{PaO}_{2}<8 \mathrm{kPa}\right.$ and $\mathrm{PaCO}_{2}$ $>6.7 \mathrm{kPa}$ ). Seventeen patients completed the study; nine (five men) of mean (SE) age 64 (2.3) years and forced expiratory volume in one second $\left(\mathrm{FEV}_{1}\right) 0.76(0.02)$ l received NIPPV and eight (four men) of mean (SE) age 61.9 (2.7) years and $\mathrm{FEV}_{1} 0.77(0.09) 1$ received doxapram. The study was approved by the Glasgow West ethical committee and informed consent was obtained.

STUDY DESIGN

Blood gas tensions were measured on admission. Patients were then started on routine treatment with oxygen (1-3 $1 / \mathrm{min}$ by nasal cannulae), steroids, antibiotics, and nebulised or intravenous bronchodilators. One hour later arterial blood gas tensions were repeated. If the values were static or deteriorating, the patient was randomised to receive either nasal ventilation or doxapram. Oxygen was continued at the same rate as before randomisation. NIPPV was administered via a pressure cycled machine (Ventimate; Thomas Respiratory Systems, London, UK). Ventilation pressure was set at 


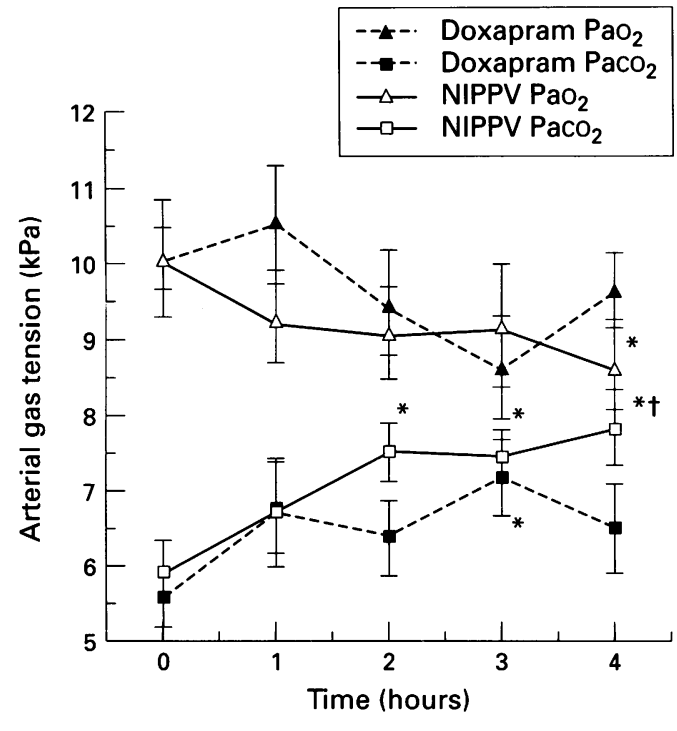

Figure 1 Mean (SE) changes in $\mathrm{PaO}_{2}$ and $\mathrm{PaCO}_{2}$ in patients admitted with acute respiratory failure treated with either intravenous doxapram $(n=8)$ or nasal intermittent positive pressure ventilation (NIPPV, $n=9)$. $* p<0.05$ compared with baseline. $\dagger p<0.05$ NIPPV compared with doxapram.

14-18 $\mathrm{cm} \mathrm{H}_{2} \mathrm{O}$ and oxygen enriched air was delivered using nasal pillows or a tight fitting nasal mask. Doxapram was given by intravenous infusion using the manufacturer's protocol $(4 \mathrm{mg} / \mathrm{min}$ for $15 \mathrm{minutes}, 3 \mathrm{mg} / \mathrm{min}$ for 30 minutes, $2 \mathrm{mg} / \mathrm{min}$ for 60 minutes, and $1.5 \mathrm{mg} / \mathrm{min}$ as maintenance dose). Arterial blood gas tensions were checked hourly for the first four hours. The protocol was modified later so that if there was continued deterioration on either NIPPV or doxapram the alternative was added at four hours.

\section{DATA ANALYSIS}

Arterial blood gas tensions were compared using ANOVA. pH was converted to $\mathrm{H}^{+}$concentration, compared by ANOVA, and reconverted to $\mathrm{pH}$ expressed as the geometric mean $+95 \%$ confidence intervals. $p$ values of less than 0.05 were regarded as significant.

\section{Results}

At entry the baseline blood gas tensions were similar in the two groups. The changes in arterial blood gas tensions during the four hour assessment period are shown in fig 1 . The mean (SE) baseline $\mathrm{PaO}_{2}$ of $5.9(0.4) \mathrm{kPa}$ improved on NIPPV to a maximum value of $8.1(0.6)$ $\mathrm{kPa}(\mathrm{p}<0.05)$ which was maintained at four hours. The baseline $\mathrm{PaCO}_{2}$ of 10.1 (0.4) $\mathrm{kPa}$ also improved on NIPPV to $8.7(0.6) \mathrm{kPa}$ $(p<0.05)$, and the improvement was maintained at four hours.

In patients receiving doxapram the mean (SE) baseline $\mathrm{PaO}_{2}$ of $5.6(0.4) \mathrm{kPa}$ improved on treatment to a maximum value of $7.3(0.5)$ $\mathrm{kPa}(\mathrm{p}<0.05)$, but this was not maintained at four hours. The baseline $\mathrm{PaCO}_{2}$ of 10.1 (0.8) $\mathrm{kPa}$ did not improve on treatment. At four hours the change in $\mathrm{PaO}_{2}$ was significantly greater in the NIPPV group than in the doxapram group.

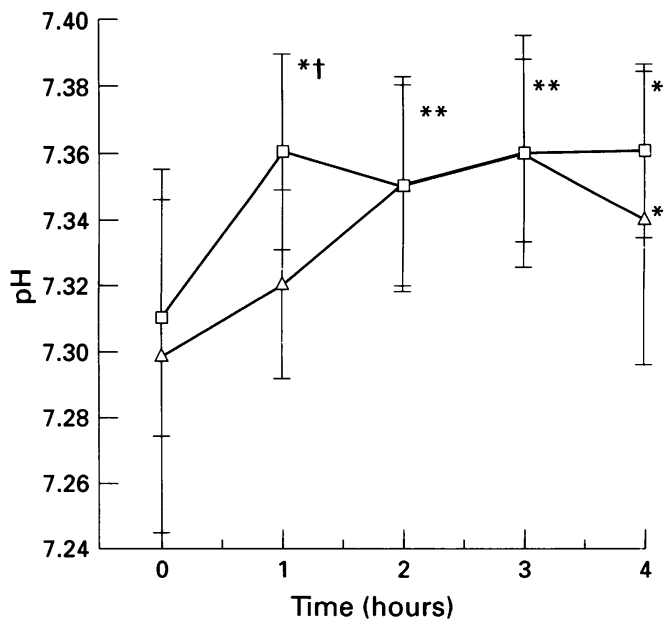

Figure 2 Changes in arterial $p H$ in patients admitted with acute respiratory failure following treatment with intravenous doxapram $(\triangle n=8)$ or NIPPV $(\square, n=9)$. Geometric mean and $95 \%$ confidence intervals are shown. $* p<0.05$ compared with baseline. $\dagger p<0.05$ NIPPV compared with doxapram.

Neither group was severely acidotic (mean pH 7.30 in the doxapram group and 7.31 in the NIPPV group). Changes in $\mathrm{pH}$ during the study are shown in fig 2 .

All patients tolerated NIPPV but doxapram was added in one because of drowsiness at four hours. All patients who received NIPPV were discharged home.

Three patients in the doxapram group died after four hours but within the first 24 hours. During the first four hours their gas tensions had continued to deteriorate despite treatment and a decision was made not to offer full mechanical ventilation. After these three deaths the protocol was altered to allow the addition of the alternative form of respiratory support. Two further patients on doxapram had NIPPV added at four hours because their clinical condition and blood gas tensions were deteriorating $\left(\mathrm{PaCO}_{2}\right.$ rose from 8.5 to 8.7 and $\mathrm{pH}$ fell from 7.31 to 7.28). Their arterial blood gas tensions improved on NIPPV (mean $\mathrm{PaO}_{2}$ improved from $7.6 \mathrm{kPa}$ to $11.3 \mathrm{kPa}$ and mean $\mathrm{PaCO}_{2}$ improved from $8.7 \mathrm{kPa}$ to $8.3 \mathrm{kPa}$ ). They both survived to discharge.

\section{Discussion}

This study has shown that non-invasive nasal ventilation produces an immediate improvement in gas exchange in patients with ventilatory failure complicating an exacerbation of COPD, and that this improvement is maintained for at least four hours. It is possible that the fractional inspired oxygen $\left(\mathrm{FIO}_{2}\right)$ was altered by the insertion of the nasal mask circuit but the fall in $\mathrm{PaCO}_{2}$ with NIPPV suggests an improvement in ventilation.

In contrast, on doxapram an initial improvement in $\mathrm{PaO}_{2}$ was not maintained. There are three explanations for this late deterioration. Firstly, the prescribing guidelines recommending a high initial loading dose followed by a reduction may be inappropriate. Secondly, although matched and randomised, the doxapram group may have simply 
deteriorated more rapidly. Thirdly, and most likely, with the respiratory muscles already working at near capacity a stimulant may offer only temporary benefit until fatigue supervenes. In contrast, assisted ventilation may offload the respiratory muscles.

Between four and 24 hours three patients in the doxapram group died. Two further patients who deteriorated in the doxapram group were treated successfully by the addition of NIPPV. One patient in the NIPPV group received doxapram, resulting in better cooperation with the nasal ventilation. While it might have been possible to alter ventilator settings rather than add doxapram, we would suggest that the addition of doxapram might improve acceptance of NIPPV, particularly if, as here, the patient was drowsy.

Despite the lack of evidence for successful treatment of ventilatory failure using doxapram in patients with acute infective exacerbations of COPD, it is widely used for this purpose. In contrast, there is good evidence for the effectiveness of NIPPV in this situation. ${ }^{4-6} \mathrm{We}$ have now shown in a small study that NIPPV is more effective than doxapram in these patients. We suggest that doxapram is reserved for patients who are centrally sedated or are unable to tolerate nasal ventilation.

1 Jeffrey AA, Warren PM, Flenley DC. Acute hypercapnic respiratory failure in patients with chronic obstructive lung respiratory failure in patients with chronic obstructive lung disease: risk fact

2 Hirsh K, Wang SC. Selective respiratory stimulating action of doxapram compared to pentylenetetrazol. $\mathcal{f}$ Pharmacol

Exp Ther 1974;189:1-11.
3 Calverley PMA, Robson RHG, Wraith PK, Prescott LF, Flenley DC. The ventilatory effects of doxapram in normal man. Clin Sci 1983;65:65-9.

4 Elliott MW, Steven MH, Phillips GD, Branthwaite MA, Non-invasive mechanical ventilation for acute respiratory failure. $B M \mathscr{F}$ 1990;300:358-60.

5 Brochard L, Isabey D, Piquet J, Amaro P, Mancebo J, Messadi $\mathrm{A}$, et al. Reversal of acute exacerbation of chronic obstructive lung disease by inspiratory assistance with face mask. $N$ Engl f Med 1990;323:1523-30.

6 Bott J, Caroll MP, Conway JH, Keilty SEJ, Ward EM, Brown $\mathrm{AM}$, et al. Randomised controlled trial of nasal ventilation in acute ventilatory failure due to chronic obstructive airways disease. Lancet 1993;341:1555-7.

7 Elliott MW, Simonds AK, Carroll MP, Wedzicha JA, Branthwaite MA. Domiciliary nocturnal nasal intermittent positive pressure ventilation in hypercapnic respiratory failure due to chronic obstructive lung disease Thorax 1992;47: 342-8. 